\title{
Elevated expression of KIF18A enhances cell proliferation and predicts poor survival in human clear cell renal carcinoma
}

\author{
QI CHEN, BIN CAO, NING NAN, YU WANG, XU ZHAI, YOUFANG LI and TIE CHONG \\ Department of Urology, The Second Affiliated Hospital of Xi'an Jiaotong University, Xi'an, Shaanxi 710004, P.R. China
}

Received December 26, 2014; Accepted February 18, 2016

DOI: 10.3892/etm.2016.3335

\begin{abstract}
The function of kinesin family member $18 \mathrm{~A}$ (KIF18A) in human renal cell carcinoma (RCC) is unclear. The purpose of the current study was to determine the expression and prognostic significance of KIF18A in RCC. Specimens from 273 RCC patients undergoing nephrectomies were studied. Expression of KIF18A mRNA was examined by reverse transcription-polymerase chain reaction (RT-PCR) or quantitative PCR, and the expression of KIF18A protein was examined by immunohistochemistry and western blotting. The expression of KIF18A in clear-cell RCC cell lines was decreased using small interfering RNA targeting KIF18A, and increased by transfection with KIF18A cDNA. The proliferative ability of RCC cells in vitro and in vivo was detected by WST-1 assay and an animal xenograft model with BALB/c nude mice, respectively. The association between KIF18A expression and overall survival was calculated using Kaplan-Meier analysis. The results showed that KIF18A expression was significantly increased in RCC tissues compared with normal kidney tissues. The level of KIF18A expression was significantly associated with tumor stage, histological grade, metastasis and tumor size. Moreover, KIF18A increased the proliferation of RCC cells in vitro and in vivo. KIF18A expression was upregulated in RCC and enhanced the proliferation of RCC cells. Therefore, it appears that KIF18A plays a key role in the carcinogenesis and progression of RCC, and is a novel candidate prognostic marker for RCC patients. Furthermore, silencing KIF18A expression may serve as a new therapeutic strategy against RCC.
\end{abstract}

\section{Introduction}

Renal cell carcinoma (RCC) is a particularly lethal genitourinary neoplasm, with an incidence of 5-10 per 100,000 and

Correspondence to: Dr Tie Chong, Department of Urology, The Second Affiliated Hospital of Xi'an Jiaotong University, 157 West 5th Road, Xi'an, Shaanxi 710004, P.R. China

E-mail: tiechong6687@163.com

Key words: renal cell carcinoma, kinesin family member 18A, prognosis accounting for 2-3\% of all malignancies (1). Clear-cell renal cell carcinoma (ccRCC) is the most common histological subtype of human RCC (2), with which $\sim 40,000$ patients are diagnosed annually in the US (1) and 650,000 patients are diagnosed annually in China (3). In $\sim 30 \%$ of patients who present with localized RCC at the time of diagnosis, distant metastases will develop within 3 years (4). RCC is resistant to chemotherapy and radiotherapy (5). The 5-year survival rate amongst patients with early stage RCC is $\sim 55 \%$; however, once RCC develops, the prognosis of advanced RCC is extremely poor and the 5 -year survival rate for advanced RCC is $10 \%$ (6). Although numerous genetic and epigenetic alterations have been shown to be correlated with RCC (7), the mechanism of carcinogenesis and progression is poorly understood. Therefore, a reliable biomarker is required to predict early metastasis and to serve as a novel therapeutic target for RCC.

Kinesin family member 18A (KIF18A) has important roles in various cellular processes, including motility, cell division, microtubule dynamics and organelle transportation (8-11). During mitosis, KIF18A is concentrated at the plus ends of microtubules and facilitates microtubule depolymerization (12). KIF18A attenuates chromosome oscillation by depolymerizing microtubules during cellular division, and therefore enhances chromosome congregation (10). In addition, loss of KIF18A activity influences chromosome segregation and leads to chromosome instability (13). It has been reported that KIF18A suppresses the movement of kinetochores to regulate mitotic chromosome alignment in the pre-anaphase state of the cell cycle (14). Furthermore, it has been confirmed that KIF18A plays critical roles in the modulation of motility and mitosis, suggesting that KIF18A acts as a critical regulator in cell transformation and carcinogenesis (15). Notably, several studies have indicated that KIF18A is involved in breast carcinogenesis and colorectal cancer progression $(16,17)$. Moreover, KIF18A has also been identified as a potential biomarker of lung cancer and cholangiocarcinoma using proteomic analysis $(18,19)$.

The expression and function of KIF18A in RCC is currently unclear. To the best of our knowledge, there have been no previous reports regarding the role of KIF18A in carcinogenesis, progression and prognostication in patients with RCC. In the current study, the expression of KIF18A and the effect of KIF18A on proliferation in human RCC were determined. KIF18A expression levels were compared in clinical specimens of RCC and normal kidney tissues, and the effects of 
transfection with KIF18A cDNA or small interfering RNA (siRNA) on the proliferation of ccRCC cells were determined in vitro and in vivo. The potential of KIF18A as an independent prognostic marker for patients with RCC, and as a therapeutic target for the treatment of RCC were also evaluated.

\section{Materials and methods}

Patients and specimens. This study included 273 consecutive patients who underwent radical or partial nephrectomies in the Department of Urology at The Second Affiliated Hospital of Xi'an Jiaotong University (Xi'an, China) between May 2005 and May 2012. The histological cell type of all resections was determined by two experienced pathologists and all specimens were confirmed to be ccRCC. The baseline clinical and pathological data and follow-up outcomes were recorded. TNM stage and Fuhrman grading were identified according to the TNM system of the 2010 American Joint Committee on Cancer (AJCC) (20) and the 2004 World Health Organization (WHO) criteria (21), respectively. RCC tissues and corresponding normal healthy kidney tissues were collected immediately after surgical resection, and all tissue specimens were formalin-fixed and paraffin-embedded; tissues were also maintained in liquid nitrogen until protein or RNA extraction. The study was approved by the Ethics Committee of the Second Affiliated Hospital of Xi'an Jiaotong University.

Cell culture. Four ccRCC cell lines (ACHN, A498, Caki-1 and Caki-2) were used in this study, all of which were purchased from the American Type Culture Collection (ATCC; Rockville, MD, USA) and were cultured in complete medium (RPMI-1640) supplemented with $10 \%$ fetal bovine serum (Gibco; Thermo Fisher Scientific, Inc., Waltham, MA, USA), streptomycin $(100 \mathrm{mg} / \mathrm{ml})$, penicillin $(100 \mathrm{U} / \mathrm{ml})$, 25 mM 4-(2-hydroxyethyl)-1-piperazineethanesulphonic acid (HEPES) and $2 \mathrm{mM}$ glutamine. All RCC cell lines were maintained as monolayers in a $10-\mathrm{cm}$ plastic dish and cultured in an incubator with a humidified atmosphere containing $5 \% \mathrm{CO}_{2}$ at $37^{\circ} \mathrm{C}$.

Animal xenograft experiment. BALB/c nude mice $(\mathrm{n}=30$; male; 4 weeks old; weight, $40-50 \mathrm{~g}$ ) were obtained from the Experimental Animal Center of Xi'an Jiaotong University, and housed in a specific pathogen-free environment with temperature maintained from $26-28^{\circ} \mathrm{C}$ and a humidity of $30-40 \%$ with a 12 -h dark:light cycle and access to food and water, supplied by the Experimental Animal Center of Xi'an Jiaotong University. The mice were randomly divided into ACHN, A498, Caki-1 and Caki-2 groups, each of which was further divided into two groups with 15 mice in each group: Control and KIF18A vector groups. The control group received untreated RCC cells, and the KIF18A group received RCC cells transfected with KIF18A cDNA, as described later. A total of $3 \times 10^{8} \mathrm{RCC}$ cells were injected into the back region of each mouse and the mice were fed continuously for 5 weeks. The volume of each xenograft was recorded once a week. At the end of the 5-week period, the mice were sacrificed under deep anesthesia, and the final volume of each xenograft was measured. Xenograft volume was calculated using the following formula: $v=a b^{2} \pi / 6$, where a represents the longest diameter and $\mathrm{b}$ represents the diameter perpendicular to the longest diameter of the tumor.

Proliferation assay. The proliferative ability of the RCC cells was evaluated in vitro using the WST-1 assay. Untransfected RCC cell lines and RCC cells transfected with KIF18A cDNA or siRNA, as described later, were used. Exponentially growing cells $[(2,000 \mathrm{RCC}$ cells with $100 \mu 1$ complete medium(Sigma-Aldrich, St. Louis, MO, USA)] were seeded into 96-well microtiter plates. Following continuous incubation for 24, 48 and $72 \mathrm{~h}, 10 \mu \mathrm{l}$ WST-1 (Roche Diagnostics, $\mathrm{GmbH}$, Penzberg, Germany) was added to each well, and the RCC cells were cultured for an additional $2 \mathrm{~h}$. The absorbance, which represents the RCC cell count in each well, was analyzed with a microculture plate reader (Immunoreader NJ-2000, Nihon Intermed Co., Ltd., Tokyo, Japan) at $450 \mathrm{~nm}$.

Reverse transcription-polymerase chain reaction (RT-PCR) and quantitative PCR (qPCR). The levels of KIF18A expression in RCC and corresponding non-cancerous tissues were detected using RT-PCR and RT-qPCR assays. Total RNA was extracted with TRizol reagent (Invitrogen; Thermo Fisher Scientific Thermo Fisher Scientific, Inc.) and reverse transcription was performed using a first-strand cDNA synthesis kit (Amersham; GE Healthcare, Chalfont, UK) according to the manufacturer's protocol. The primer sequences of KIF18A were determined using Primer Premier 5.0 software (Premier Biosoft, Palo Alto, CA, USA) and glyceraldehyde-3-phosphate hydrogenase (GAPDH) was used as a control. The primer sequences for KIF18A were as follows: 5'-AAAAAGTGG TAGTTTGGGCTGA-3' (sense); and 5'-CTTTCAAGGGAG ATGGCATTAG-3' (antisense). The primer sequences for GAPDH were as follows: 5'-ATCAAGAAGGTGGTGAAG CAG-3' (sense); and 5'-TGGAGGAGTGGGTGTCGC-3' (antisense). Products were amplified by PCR using a TaqMan Universal PCR Master Mix kit (Applied Biosystems; Thermo Fisher Scientific, Inc.) and data was obtained and analyzed with a LightCycler 480 (Roche Diagnostics, Indianapolis, IN, USA). All RT reactions were performed in triplicate, and experimental procedures of qPCR were based on MIQE guidelines (22). The relative expression levels determined by the $2^{-\Delta \Delta c q}$ method (23).

Immunohistochemistry. All sections were dewaxed with xylene and rehydrated in graded alcohols. Antigen retrieval was conducted in citrate buffer and the sections were then washed in phosphate-buffered saline (PBS). Endogenous peroxidase activity was blocked with $3 \%$ hydrogen peroxide for $20 \mathrm{~min}$. Then, sections were incubated with $10 \%$ goat serum (Thermo Fisher Scientific, Inc.) at room temperature for $30 \mathrm{~min}$ to block non-specific binding. Sections were incubated with rabbit polyclonal anti-KIF18A antibody (cat no. 19245; 1:1,1000; Proteintech ${ }^{\mathrm{TM}}$ Group, Inc., Chicago, IL, USA) for $16 \mathrm{~h}$ at $4^{\circ} \mathrm{C}$, washed with PBS, incubated with biotinylated goat anti-rabbit antibody (cat no. E0432; 1:2,000; Dako, Glostrup, Denmark) for $2 \mathrm{~h}$ at $37^{\circ} \mathrm{C}$, and stained with 3,3'-diaminobenzidine tetrahydrochloride.

Western blot analysis. Total protein was isolated from RCC tissues, normal kidney tissues or RCC cells using lysis buffer 
Table I. Characteristics of RCC patients and KIF18A protein and mRNA expression detected by immunohistochemistry and reverse transcription-quantitative polymerase chain reaction, respectively.

\begin{tabular}{|c|c|c|c|c|c|c|c|c|}
\hline \multirow[b]{2}{*}{ Characteristic } & \multirow[b]{2}{*}{$\mathrm{n}$} & \multicolumn{4}{|c|}{ KIF18A protein } & \multirow[b]{2}{*}{ P-value } & \multirow[b]{2}{*}{ KIF18A mRNA } & \multirow[b]{2}{*}{ P-value } \\
\hline & & - & + & ++ & +++ & & & \\
\hline Renal cell carcinoma & 273 & 29 & 126 & 72 & 46 & & $2.30 \pm 0.29$ & \\
\hline $\begin{array}{l}\text { Non-cancerous renal } \\
\text { tissue }\end{array}$ & 182 & 139 & 32 & 11 & 0 & $<0.05$ & $0.62 \pm 0.08$ & $<0.05$ \\
\hline Gender & & & & & & $>0.05$ & & $>0.05$ \\
\hline Male & 149 & 17 & 69 & 39 & 24 & & $2.27 \pm 0.28$ & \\
\hline Female & 124 & 12 & 57 & 33 & 22 & & $2.33 \pm 0.29$ & \\
\hline Age & & & & & & $>0.05$ & & $>0.05$ \\
\hline$<60$ years & 152 & 16 & 69 & 42 & 25 & & $2.30 \pm 0.26$ & \\
\hline$\geq 60$ years & 121 & 13 & 57 & 30 & 21 & & $2.28 \pm 0.28$ & \\
\hline Tumor size & & & & & & $<0.05$ & & $<0.05$ \\
\hline$\leq 7 \mathrm{~cm}$ & 147 & 23 & 103 & 14 & 7 & & $1.53 \pm 0.21$ & \\
\hline$>7 \mathrm{~cm}$ & 126 & 6 & 23 & 58 & 39 & & $3.18 \pm 0.35$ & \\
\hline Histological grade & & & & & & $<0.05$ & & $<0.05$ \\
\hline G1 & 145 & 23 & 97 & 21 & 4 & & $1.22 \pm 0.18$ & \\
\hline G2 & 90 & 6 & 29 & 46 & 9 & & $2.57 \pm 0.29$ & \\
\hline G3 & 38 & 0 & 0 & 5 & 33 & & $5.88 \pm 0.68$ & \\
\hline Tumor stage & & & & & & $<0.05$ & & $<0.05$ \\
\hline I & 147 & 23 & 103 & 14 & 7 & & $1.34 \pm 0.15$ & \\
\hline II & 47 & 4 & 17 & 21 & 5 & & $2.30 \pm 0.29$ & \\
\hline III & 44 & 2 & 5 & 16 & 21 & & $3.64 \pm 0.41$ & \\
\hline IV & 35 & 0 & 1 & 21 & 13 & & $4.59 \pm 0.52$ & \\
\hline Metastasis & & & & & & $<0.05$ & & $<0.05$ \\
\hline Absent & 242 & 29 & 125 & 54 & 34 & & $1.98 \pm 0.22$ & \\
\hline Present & 31 & 0 & 1 & 18 & 12 & & $4.76 \pm 0.53$ & \\
\hline
\end{tabular}

Protein expression is shown as staining intensity, and mRNA expression is shown as mean \pm standard deviation. RCC, renal cell carcinoma; KIF18A, kinesin family member 18A.

(cat no. ab152163, Abcam, Cambridge, UK). The protein concentration was measured using a bicinchoninic acid (BCA) protein assay kit (Pierce Biotechnology, Inc., Rockford, IL, USA). A $100-\mu$ m quantity of total protein was separated by $10 \%$ sodium dodecyl sulfate-polyacrylamide gel electrophoresis and transferred to a polyvinylidene difluoride membrane (Amersham; GE Healthcare). The membrane was then probed for $2 \mathrm{~h}$ at $37^{\circ} \mathrm{C}$ with rabbit polyclonal anti-KIF18A antibody (cat no. ab72416; 1:1,000; Abcam) with anti- $\beta$-actin monoclonal antibody (cat no. ab8226; 1:5,000; Abcam) as a loading control. Next, membranes were incubated with goat anti-rabbit IgG H\&L (HRP) (cat no. ab6721; 1:5,000; Abcam) at $37^{\circ} \mathrm{C}$ for $2 \mathrm{~h}$ ). Finally, the immune reaction complexes were visualized using an ECL Plus Western Blotting Detection Reagents (Amersham; GE Healthcare).

Cell transfection. siRNA oligonucleotide sequences targeting KIF18A were designed using siDirect software (sidirect2.rnai.jp). The four ccRCC cell lines were transiently transfected with the oligonucleotides using Lipofectamine 2000 (Invitrogen; Thermo Fisher Scientific Inc.) according to the manufacturer's protocol. Moreover, an expression vector (pcDEF3; Sigma-Aldrich) containing full-length cDNA for KIF18A was also used to stably transfect the 4 RCC cell lines using Lipofectamine 2000. Monoclonal RCC cells were collected with G418 (Sigma-Aldrich) and transfection efficiency was evaluated by western blot analysis.

Statistical analysis. Statistical calculations were carried out using SPSS software (version 19.0; IBM SPSS, Armonk, NY, USA). Differences between numerical variables were calculated using the Student's t-test and the results are presented as the mean \pm standard deviation (SD), while categorical variables were analyzed using the $\chi^{2}$ test. Survival curves were established using the Kaplan-Meier method. All experiments were performed in triplicate. All P-values were two-sided and statistical significance was set at $\mathrm{P}<0.05$.

\section{Results}

Patient clinical characteristics. Data were collected from 273 patients with RCC (149 males and 124 females) and 

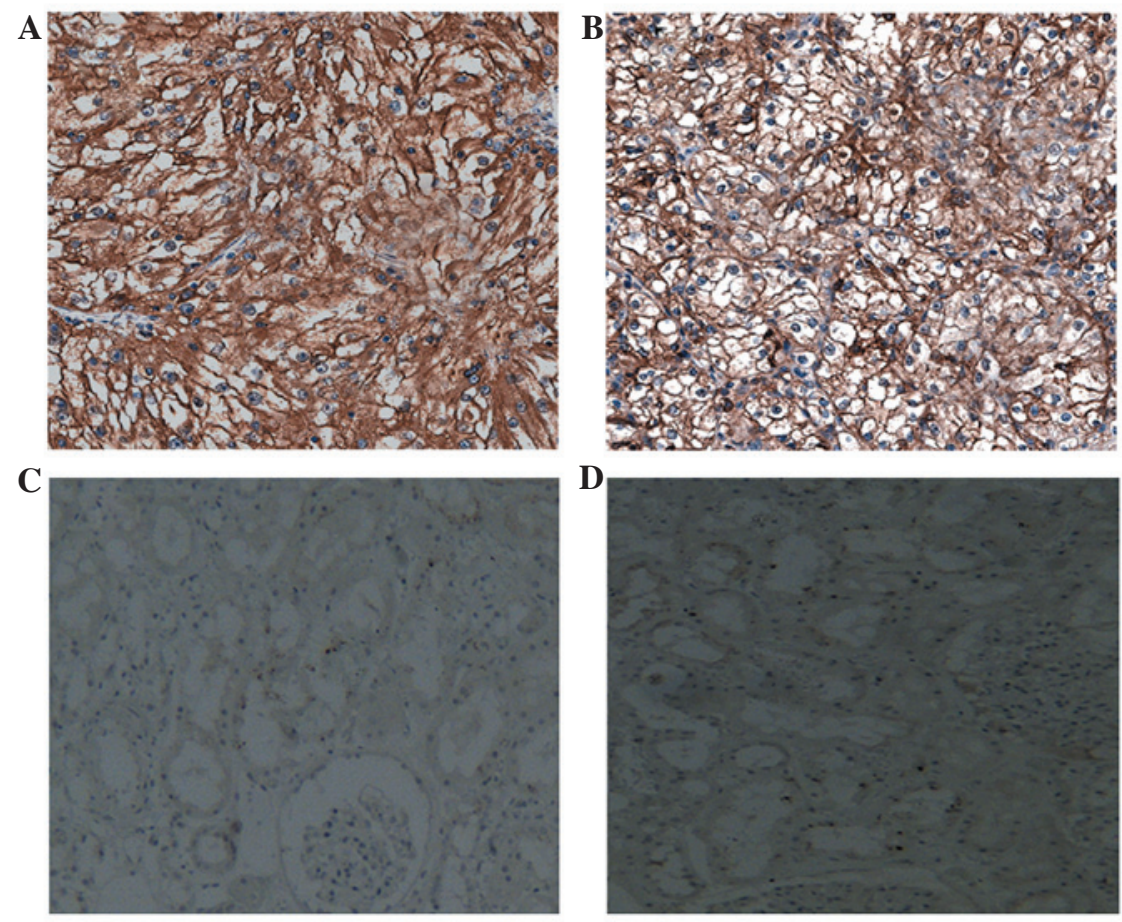

Figure 1. KIF18A expression is highly increased in (A and B) human RCC tissues compared with those in (C and D) normal kidney tissues, as indicated by immunohistochemical staining. Magnification, x400. KIF18A, kinesin family member 18A; RCC, renal cell carcinoma.

182 corresponding normal renal tissues. The ages of the patients ranged from 41 to 87 years (median, 65 years), and the tumor diameter ranged from 0.7 to $23.8 \mathrm{~cm}$ (median, $4.2 \mathrm{~cm}$ ). Among the 273 RCC patients, 147 had stage I, 47 had stage II, 44 had stage III and 35 had stage IV disease. RCC was localized in 242 patients, and 31 patients had distant metastases at the time of diagnosis. Moreover, 21 patients with RCC had previously been treated with radical nephrectomies on the contralateral side. All patient clinical characteristics are shown in Table I.

KIF18A protein expression in RCC. The expression of KIF18A in human RCC and normal kidney tissues was examined using immunohistochemistry. The expression of KIF18A protein was observed to be upregulated significantly in RCC tissues compared with the levels in corresponding normal kidney tissues (Fig. 1). KIF18A expression was detected in 244/273 RCC tissues (89.4\%), but only 43/182 (23.6\%) normal kidney tissues. The association between the expression of KIF18A protein and various clinicopathologic characteristics was investigated; a significant association existed between increased KIF18A expression and high tumor stage, high histological grade, metastasis and large tumor size $(\mathrm{P}<0.05)$. Neither of the other characteristics, age and gender, showed a significant correlation with the expression of KIF18A protein (Table I). Together, these findings indicate that upregulation of KIF18A might be involved in the carcinogenesis and development of human RCC.

KIF18A expression detected by RT-PCR, RT-qPCR and western blot analysis. To confirm the expression levels of KIF18A protein in human RCC as indicated by immunohistochemistry, the expression of KIF18A in RCC and normal kidney tissues was also evaluated by RT-PCR, RT-qPCR and western blot analysis. The level of KIF18A expression was analyzed with reference to an internal control; the results suggested that KIF18A expression was upregulated significantly in the RCC tissues compared with the corresponding normal kidney tissues. Moreover, the expression levels of KIF18A mRNA were comparable with the levels detected by immunohistochemistry (Table I). Representative results for four pairs of RCC and corresponding normal kidney tissues are shown in Fig. 2.

Effect of KIF18A on the proliferation of RCC cells. KIF18A expression was stably increased by transfecting a vector containing the full-length cDNA of KIF18A into ACHN, A498, Caki-1 and Caki-2 cell lines. In addition, KIF18A expression was also decreased using siRNA technology. The transfection efficiency was evaluated by western blotting. The expression of KIF18A protein was significantly increased by the KIF18A vector insert and decreased by the siRNA (Fig. 3A). The effect of KIF18A on the proliferative ability of RCC cells in vitro was analyzed by WST-1 assay. RCC cells with a high level of KIF18A expression had significantly increased proliferation compared with untreated control cells. By contrast, RCC cells with a low level of KIF18A expression had lower proliferative ability (Fig. 3B). These results were also confirmed in vivo by animal xenograft experiments with BALB/c nude mice, which showed that KIF18A transfection increased xenograft volume in the four cell lines (Fig. 3C).

Prognostic significance of KIF18A expression in RCC. Since significant correlations were demonstrated between KIF18A expression and clinical stage, pathological grade, and tumor metastasis in human RCC, it was further evaluated whether or not KIF18A could be regarded as a novel prognostic marker in 
A

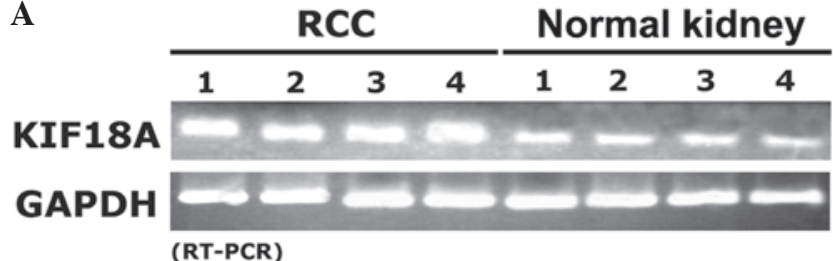

B

\begin{tabular}{ccccccccc}
\multicolumn{4}{c}{ RCC } & & \multicolumn{3}{c}{ Normal kidney } \\
\hline 1 & 2 & 3 & 4 & & 1 & 2 & 3 & 4
\end{tabular}

\section{KIF18A}

\section{$\beta$-actin}

C

(Western blot )

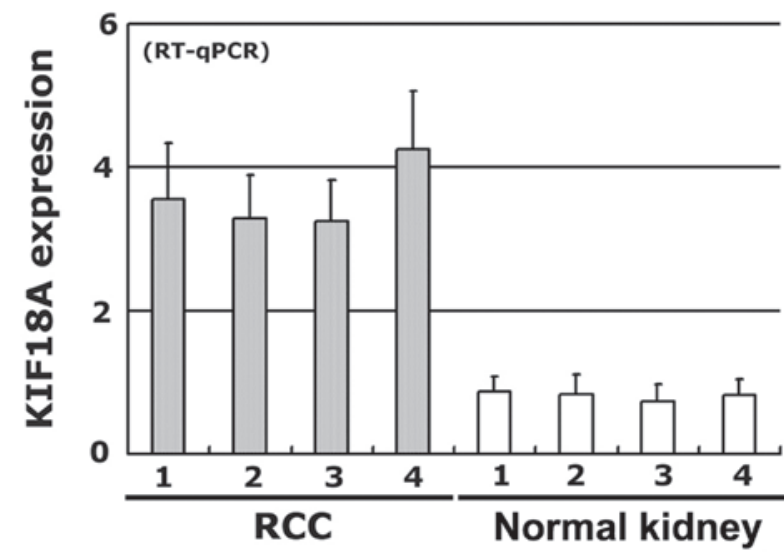

Figure 2. KIF18A expression detected by (A) RT-PCR, (B) western blotting and (C) RT-qPCR in RCC and corresponding normal kidney tissues from 4 representative patients. All experiments were performed in triplicate; the error bars represents standard deviation. KIF18A, kinesin family member 18A; RCC, renal cell carcinoma; GAPDH, glyceraldehyde-3-phosphate hydrogenase; RT-PCR, reverse transcription-polymerase chain reaction; RT-qPCR, RT-quantitative PCR.

human RCC. Kaplan-Meier analysis was carried out to calculate the association between KIF18A expression and overall survival in RCC patients. The overall survival of patients with RCC was found to be significantly different between the high and low KIF18A expression groups $(\mathrm{P}<0.05)$. After 10 years of follow-up, the overall survival time of RCC patients who expressed a low level of KIF18A expression by immunohistochemistry $(-$ or +$)$ was significantly longer compared with that of the patients who expressed a high level of KIF18A (++ or +++ ) (Fig. 4). These findings indicate that high KIF18A expression might be an independent marker to predict poor prognosis in patients with RCC.

\section{Discussion}

Various motor proteins of the kinesin family have emerged as targets for chemotherapeutic interventions of malignancies due to the crucial effects on cell mitosis (24-26). KIF18A, a member of the kinesin family, plays a key role in the carcinogenesis and progression of tumors $(14,15)$. A recent study indicated that the expression of KIF18A is significantly upregulated and correlated with aggressive characteristics in patients with hepatocellular carcinoma (27). The same study also suggested the possibility that KIF18A can serve as a prognostic marker
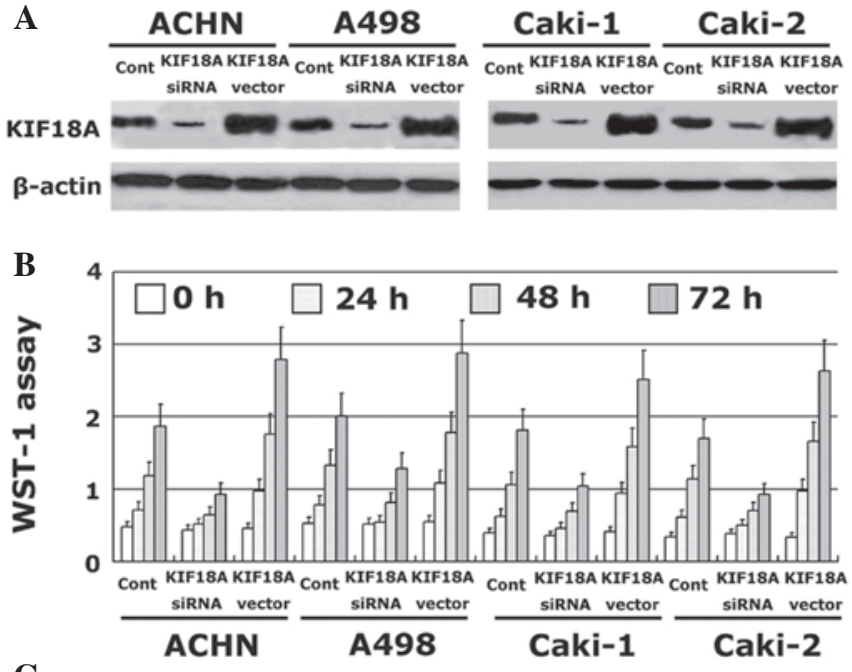

C

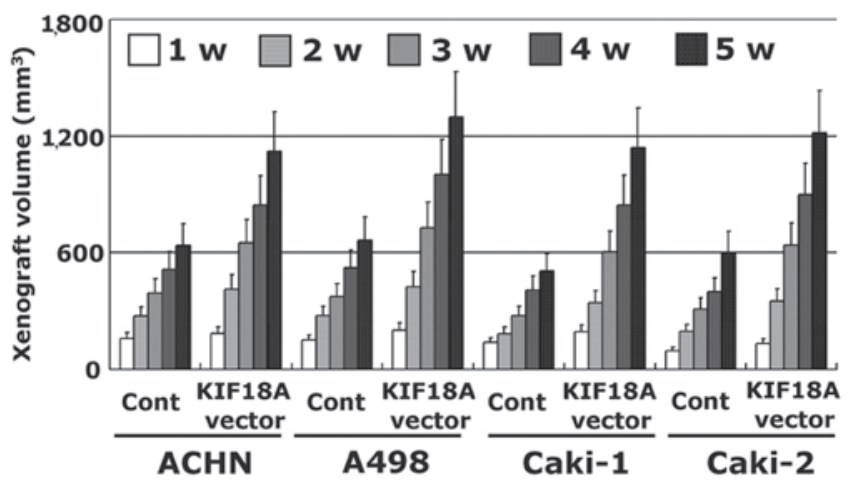

Figure 3. Effect of KIF18A on the proliferation of RCC cells. KIF18A expression was decreased using siRNA and increased by transfection with KIF18A vector in RCC cell lines. (A) All transfections were confirmed using western blot analysis. The proliferative ability of RCC cells in vitro was detected using (B) WST-1 assay and (C) an animal xenograft study. KIF18A, kinesin family member $18 \mathrm{~A}$; RCC, renal cell carcinoma; w, week.

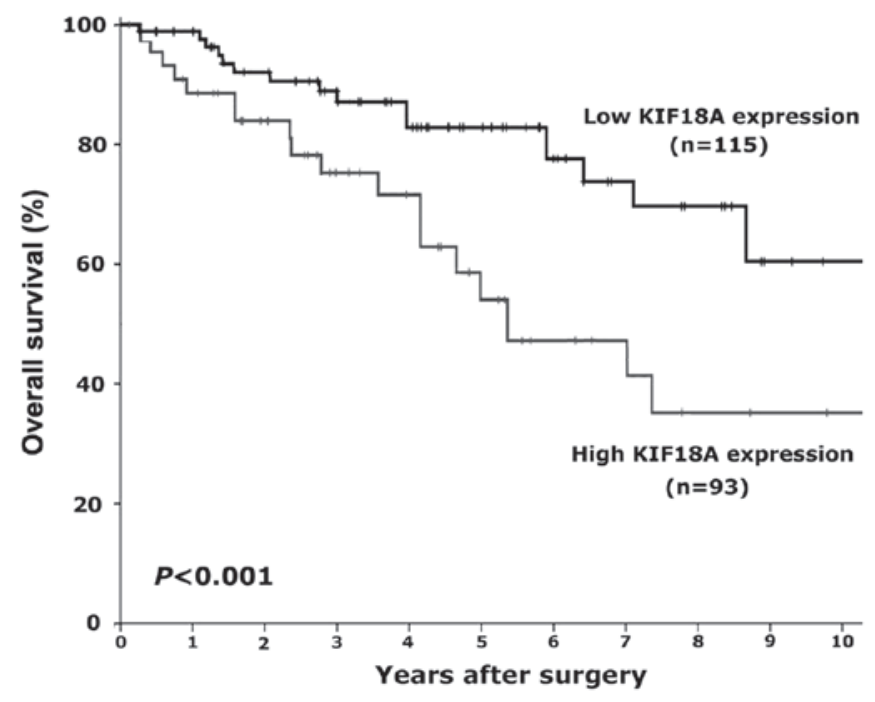

Figure 4. Kaplan-Meier analysis was performed to analyze the association between KIF18A expression and overall survival of patients with RCC. KIF18A, kinesin family member 18A; RCC, renal cell carcinoma.

in patients with hepatocellular carcinoma (27); however, the function of KIF18A in carcinogenesis is unclear. KIF18A has 
been shown to be upregulated in a colorectal cancer model, and KIF18A-deficient mice were found to be protected from carcinogenesis of colorectal cancer via inactivation of the phosphoinositide 3-kinase-Akt pathway (28). De Wever et al (29) found that KIF18A directly interacts with protein phosphatase $1 \gamma$, a serine/threonine protein phosphatase, through a conserved RVxF motif, and KIF18A participates in phosphatase-induced biological progress. Another study demonstrated that cells treated with estrogen express higher levels of KIF18A mRNA and protein, which added novel insight into the role of estrogen in the regulation of KIF18A expression (30). In addition, a study conducted by Zusev and Benayahu (31) indicated that KIF18A is involved in post-translational modifications and plays a potential role in regulating protein distribution and the co-association with cytoskeletal proteins. KIF18A has generated considerable attention with respect to carcinogenesis, and the expression of KIF18A has also been detected in tumors. The expression of KIF18A and the function of KIF18A in human RCC, however, remains elusive to date.

On the basis of a review of the literature, to the best of our knowledge, this is the first study involving KIF18A expression in RCC. In the present study, the level of KIF18A expression in human RCC was detected, and the expression of KIF18A mRNA in RCC tissues was detected by RT-PCR and RT-qPCR. The expression of KIF18A mRNA was similar to the levels of protein detected by immunohistochemistry and western blotting. KIF18A expression was significantly upregulated in RCC tissues compared with normal kidney tissues. Moreover, the expression of KIF18A was significantly correlated with tumor stage, histological grade, metastasis and tumor size. These findings demonstrate that KIF18A may act as an oncogene and plays a crucial role in the carcinogenesis and progression of human RCC. The effect of KIF18A on the proliferation of RCC cells was also evaluated. KIF18A significantly prompted the proliferation of RCC cells; a similar finding was observed in an animal xenograft experiment with BALB/C nude mice. The association between the level of KIF18A expression and overall survival of patients with RCC was further calculated using Kaplan-Meier analysis. High expression of KIF18A was found to be associated with poor prognosis of patients with RCC. Thus, the present study demonstrated that KIF18A can be considered as an independent marker to predict prognosis in RCC patients. It appears that the KIF18A gene plays an important role in carcinogenesis of the adult kidney, and a high level of KIF18A expression may enhance the progression of human RCC. Moreover, KIF18A is a novel candidate prognostic gene for RCC patients, thus raising the intriguing possibility that patients with RCC expressing a high level of KIF18A expression may be genetically predisposed to RCC. As the function of KIF18A has not been fully elucidated, it is necessary to analyze the detailed molecular mechanism of KIF18A in human RCC in future studies.

In summary, the results of the present study suggest that KIF18A expression is significantly increased in human RCC and KIF18A enhances the proliferation of RCC cells in vitro and in vivo. These findings indicate that KIF18A plays a key role in the carcinogenesis and progression of $\mathrm{RCC}$, and is a novel prognostic candidate marker for RCC patients. Silencing KIF18A expression may serve as a new therapeutic strategy against RCC.

\section{References}

1. Siegel R, Naishadham D and Jemal A: Cancer statistics, 2013. CA Cancer J Clin 63: 11-30, 2013.

2. Deng FM and Melamed J: Histologic variants of renal cell carcinoma: Does tumor type influence outcome? Urol Clin North Am 39: 119-132, 2012

3. Yang L, Parkin DM, Ferlay J, Li L and Chen Y: Estimates of cancer incidence in China for 2000 and projections for 2005. Cancer Epidemiol Biomark Prev 14: 243-250, 2005.

4. Athar U and Gentile TC: Treatment options for metastatic renal cell carcinoma: A review. Can J Urol 15: 3954-3966, 2008.

5. Janowitz T, Welsh SJ, Zaki K, Mulders P and Eisen T: Adjuvant therapy in renal cell carcinoma - past, present and future. Semin Oncol 40: 482-491, 2013.

6. Ljungberg B, Cowan NC, Hanbury DC, Hora M, Kuczyk MA, Merseburger AS, Patard JJ, Mulders PF and Sinescu IC; European Association of Urology Guideline Group: EAU guidelines on renal cell carcinoma: The 2010 update. Eur Urol 58: 398-406, 2010.

7. Bratslavsky G, Sanford T, Srinivasan R, Aprelikova O, Liu J, Quezado M, Merino M and Linehan WM: Differential genetic expression in large versus small clear cell renal cell carcinoma: Results from microarray analysis. J Cancer 2: 271-279, 2011.

8. Jordan MA and Wilson L: Microtubules as a target for anticancer drugs. Nat Rev Cancer 4: 253-265, 2004.

9. Gardner MK, Odde KJ and Bloom K: Kinesin-8 molecular motors: Putting the brakes on chromosome oscillations. Trends Cell Biol 18: 307-310, 2008

10. Du Y, English CA and Ohi R: The kinesin-8 Kif18A dampens microtubule plus-end dynamics. Curr Biol 20: 374-380, 2010.

11. Weaver LN, Ems-McClung SC, Stout JR, LeBlanc C, Shaw SL, Gardner MK and Walczak CE: Kif18A uses a microtubule binding site in the tail for plus-end localization and spindle length regulation. Curr Biol 21: 1500-1506, 2011.

12. Mayr MI, Hümmer S, Bormann J, Grüner T, Adio S, Woehlke G and Mayer TU: The human kinesin Kif18A is a motile microtubule depolymerase essential for chromosome congression. Curr Biol 17: 488-498, 2007.

13. Hartwell LH and Kastan MB: Cell cycle control and cancer. Science 266: 1821-1828, 1994.

14. Malumbres M and Barbacid M: Cell cycle, CDKs and cancer: A changing paradigm. Nat Rev Cancer 9: 153-166, 2009.

15. Mayr MI, Storch M, Howard J and Mayer TU: A non-motor microtubule binding site is essential for the high processivity and mitotic function of kinesin-8 Kif18A. PLoS One 6: e27471, 2011.

16. Zhang C, Zhu C, Chen H, Li L, Guo L, Jiang W and Lu SH: Kif $18 \mathrm{~A}$ is involved in human breast carcinogenesis, Carcinogenesis 31: 1676-1684, 2010.

17. Nagahara M, Nishida N, Iwatsuki M, Ishimaru S, Mimori K, Tanaka F, Nakagawa T, Sato T, Sugihara K, Hoon DS and Mori M: Kinesin 18A expression: Clinical relevance to colorectal cancer progression. Int J Cancer 129: 2543-2552, 2011.

18. Tooker BC, Newman LS, Bowler RP, Karjalainen A, Oksa P, Vainio H, Pukkala E and Brandt-Rauf PW: Proteomic detection of cancer in asbestosis patients using SELDI-TOF discovered serum protein biomarkers. Biomarkers 16: 181-191, 2011.

19. Rucksaken R, Khoontawad J, Roytrakul S, Pinlaor P, Hiraku Y, Wongkham C, Pairojkul C, Boonmars T and Pinlaor S: Proteomic analysis to identify plasma orosomucoid 2 and kinesin $18 \mathrm{~A}$ as potential biomarkers of cholangiocarcinoma. Cancer Biomark 12: 81-95, 2012

20. Elmore JM, Kadesky KT, Koeneman KS and Sagalowsky AI: Reassessment of the 1997 TNM classification system for renal cell carcinoma. Cancer 98: 2329-2334, 2003.

21. Hong SK, Jeong CW, Park JH, Kim HS, Kwak C, Choe G, Kim HH and Lee SE: Application of simplified Fuhrman grading system in clear-cell renal cell carcinoma. BJU Int 107: 409-415, 2011.

22. Johnson GL, Bibby DF, Wong S, Agrawal SG and Bustin SA: A MIQE-compliant real-time PCR assay for Aspergillus detection. PLoS One 7: e40022, 2012.

23. Grimholt RM, Urdal P, Klingenberg O and Piehler AP: Rapid and reliable detection of $\alpha$-globin copy number variations by quantitative real-time PCR. BMC Hematol 14: 4, 2014.

24. Rath O and Kozielski F: Kinesins and cancer. Nat Rev Cancer 12: 527-539, 2012.

25. Wood KW, Cornwell WD and Jackson JR: Past and future of the mitotic spindle as an oncology target. Curr Opin Pharmacol 1: 370-377, 2001. 
26. Huszar D, Theoclitou ME, Skolnik J and Herbst R: Kinesin motor proteins as targets for cancer therapy. Cancer Metastasis Rev 28: 197-208, 2009.

27. Liao W, Huang G, Liao Y, Yang J, Chen Q, Xiao S, Jin J, He S and Wang C: High KIF18A expression correlates with unfavorable prognosis in primary hepatocellular carcinoma. Oncotarget 5: 10271-10279, 2014.

28. Zhu H, Xu W, Zhang H, Liu J, Xu H, Lu S, Dang S, Kuang Y, Jin $\mathrm{X}$ and Wang Z: Targeted deletion of Kif 18 a protects from colitis-associated colorectal (CAC) tumors in mice through impairing Akt phosphorylation. Biochem Biophys Res Commun 438: 97-102, 2013.
29. De Wever V, Nasa I, Chamousset D, Lloyd D, Nimick M, $\mathrm{Xu}$ H, Trinkle-Mulcahy L and Moorhead GB: The human mitotic kinesin KIF18A binds protein phosphatase 1 (PP1) through a highly conserved docking motif. Biochem Biophys Res Commun 453: 432-437, 2014.

30. Zusev M and Benayahu D: The regulation of MS-KIF18A expression and cross talk with estrogen receptor. PLoS One 4: e6407, 2009.

31. Zusev M and Benayahu D: New insights on cellular distribution, microtubule interactions and post-translational modifications of MS-KIF18A. J Cell Physiol 217: 618-625, 2008 . 